\title{
Therapeutic Massage Provides Pain Relief to a Client with Morton's Neuroma: A Case Report
}

\author{
Faith Davis, BA, RMT, NCTMB, AOS
}

Private Practice, Boulder, CO, USA

Background: Morton's neuroma is a common cause of pain that radiates from between the third and fourth metatarsals and which, when symptomatic, creates sensations of burning or sharp pain and numbness on the forefoot. Many conservative and surgical interventions are employed to reduce associated pain, but not enough research has been conducted to recommend patients to any one approach as the most reliable source of pain management.

Purpose: The objective of this case report is to describe the effect of massage therapy on one woman with symptomatic Morton's neuroma.

Participant: A physically active 25-year-old female with diagnosed symptomatic Morton's neuroma who has not found relief with previous conservative intervention.

Intervention: Six session of massage therapy once weekly for 60-75 minutes focused on postural alignment and localized foot and leg treatment. The client also completed an at-home exercise each day. Change was monitored each week by the massage therapist reassessing posture and by the client filling out a pain survey based on a Visual Analog Scale.

Results: The client reported progressive change in the character of the pain from burning and stabbing before the first session to a dull, pulsing sensation after the third session. She also recorded a reduction in pain during exercise from a 5/10 to 0/10 (on a scale where 10 is extreme pain).

Conclusion: This study describes how massage therapy reduced pain from Morton's neuroma for one client; however, larger randomized control studies need to be done in order to determine the short- and long-term effects of massage therapy on this painful condition.

KEYWORDS: intermetatarsal neuroma, foot health, entrapment neuropathy, Mulder's sign, forefoot, deep transverse metatarsal ligaments

\section{INTRODUCTION}

\section{Morton's Neuroma}

Morton's neuroma is a common pathology of the foot in the United States. It is prevalent in people aged
$15-50$, but is most commonly seen in middle-aged patients $^{(1,2)}$. It occurs ten times more frequently in women than in men ${ }^{(3)}$. Sometimes called intermetatarsal neuroma, it is an entrapment neuropathy associated with compression of the common interdigital nerve beneath the transverse metatarsal ligament ${ }^{(4)}$. As a result of this entrapment, the common interdigital nerve swells distal to the deep transverse metatarsal ligament, most commonly between the third and fourth metatarsal bones ${ }^{(5)}$. Pain results when the metatarsals compress and grind the swollen nerve ${ }^{(5)}$. Not all Morton's neuromas are symptomatic (painful $)^{(1)}$, but those that are symptomatic are characterized by paraesthesia — numbness, burning, or sharp pain - on the bottom of the foot that radiates from the third and fourth metatarsals into the third and fourth phalanges. The pain and numbness caused by symptomatic Morton's neuroma can vary between irritating and debilitating, and treatments are equally variable. Interventions range from conservative to surgical. Conservative treatments include footwear changes, steroid injections, physical therapy, and topical or NSAID pain relievers ${ }^{(1,6)}$. If these fail to relieve the pain, surgery to either remove the neuroma or release the pressure from the ligament is often the next step.

\section{Anatomy and Pathology}

Morton's neuroma is not a true neuroma. A true neuroma is a proliferation of nerve axons, or a tumor of neurons. Morton's neuroma is instead a lesion which, "consists of perineural fibrosis, local vascular proliferation, edema of the endoneurium, and axonal degeneration"(7) (Figure 1). Because it is a compression neuropathy of the common interdigital nerve, some prefer the term interdigital neuritis as a more accurate representation of the problem ${ }^{(8)}$. The lesion will be referred to by its common name "Morton's neuroma" for the duration of this report.

While the exact cause of Morton's neuroma is not known, it is thought to be linked to hypermobility of the metatarsals ${ }^{(5)}$ and repetitive motions which grind the nerve bundle between the metatarsals. Anecdotal evidence suggests that Morton's neuroma may be related to crush injuries or repetitive running or jumping motions where the foot strikes with such 


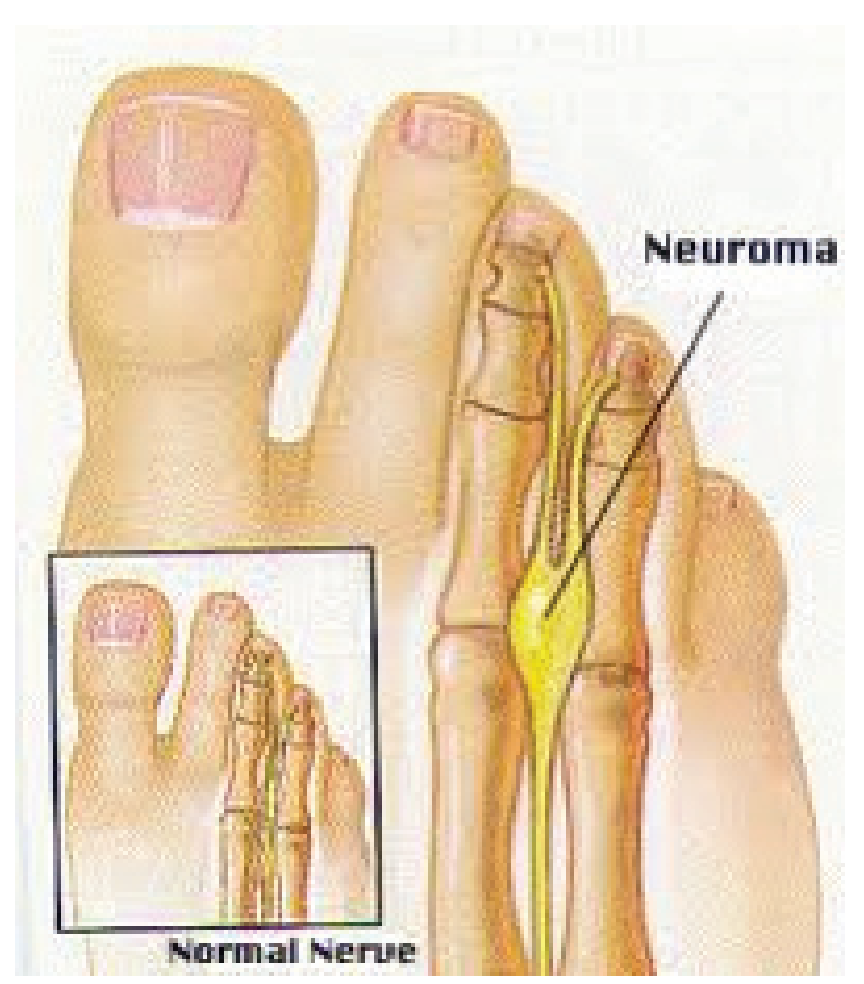

FIGURE 1. Image of Morton's Neuroma.

compressive force that the metatarsals are damaged or the nerve is in some way impinged. Damage or injury to this area of the forefoot is more susceptible to lesion formation because of the large nerve bundle created when the lateral plantar nerve and the medial plantar nerve come together at the third and fourth metatarsals ${ }^{(9)}$.

Structural misalignment and mechanical abnormalities of the forefoot may also be contributing factors in creating symptomatic Morton's neuroma ${ }^{(8)}$. One structural concern is that if the intermetatarsal ligament is lax, the interdigital nerve tissue will shift into the area between the metatarsal heads and be subject to compressive trauma ${ }^{(6)}$.

The pain caused by Morton's neuroma is typically associated with standing activity and may be linked to wearing heels or tight shoes that compress the toe box ${ }^{(9)}$. Although it does not necessarily follow a common pattern, Morton's neuroma pain is usually "triggered by walking and relieved by removing shoes and by various manipulations of the forefoot, but pain may occur at night or without apparent precipitating factors"(10).

\section{Identifying Morton's Neuroma}

The clinical test for Morton's neuroma is to "compress the foot by applying pressure to the medial and lateral aspects of the foot at the metatarsophalangeal joints," in turn putting pressure on the nerves ${ }^{(5)}$. A positive test is indicated by a sharp pain at the location of the neuroma that mimics the pain the patient normally feels ${ }^{(5)}$. Patients with Morton's neuroma may also exhibit Mulder's sign, an audible and "palpable click when pressure is applied to the sole of the foot and the metatarsals are subsequently squeezed together"(7), but this is only seen in $20 \%$ of those with symptomatic Morton's neuroma ${ }^{(8)}$. In some cases, magnetic resonance imagery (MRI) is used to identify atypical presentations, but one review suggests these presurgery MRIs may be a "waste of resources and set a dangerous medico-legal precedent"(11). Ultrasound and an X-ray can also be used to determine the size of the Morton's neuroma and to eliminate the possibility of complications in bony structures that can cause forefoot pain ${ }^{(3,6,7,8)}$.

\section{Treatment of Morton's Neuroma}

As previously mentioned, conservative treatments include changes in footwear, orthoses, metatarsal pads, taping, steroid injections, cryotherapy, physical therapy, and topical or NSAID pain relievers ${ }^{(1,2,6)}$. Initially, a person suffering from Morton's neuroma pain may try low-heeled, wide shoes or custom shoe orthoses. This can be especially effective in alleviating pain if high heels or shoes with a tight toe-box have been a source of pain. Many doctors and physical therapists recommend a period of rest and a dramatic reduction in activities that elicit pain ${ }^{(12,13,14)}$. Corticosteroid injections are another common intervention to diagnose and mitigate pain for a few weeks at a time $^{(8,9)}$. Multiple injections may create local problems including atrophy of the forefoot and blanched skin $^{(8)}$. In one case report, functional fascial taping was effectively used to manage pain associated with Morton's neuroma ${ }^{(6)}$, but this procedure must be examined in a larger study before any clear correlation can be made. Physical therapy may be focused on pain-free flexibility, strengthening, and balance exercises to combat atrophy from disuse and promote soft-tissue strength and flexibility ${ }^{(14)}$. Some acupuncturists believe Morton's neuroma can be managed through traditional Chinese medicine ${ }^{(15)}$, although this method is also lacking in available research reports or trials.

If conservative interventions fail to relieve the pain, surgery to remove the neuroma or to release pressure from the ligament may be recommended. Fifteen to twenty percent of these surgeries will not relieve the pain, and may be followed by complications such as local postsurgical infections, scar tissue, and soft tissue damage to the muscles and skin at the site of incision which impair functional foot movement, and recurring neuromas after the initial surgery ${ }^{(6,8)}$. This highlights the need for larger randomized control studies of nonsurgical treatment.

\section{Massage \& Morton's Neuroma}

In a study of 85 patients, those with symptomatic (painful) Morton's neuroma found relief by removing 
shoes and gentle self-massage of the forefoot ${ }^{(1)}$. In one review of research on the efficacy of manual therapies on many different musculoskeletal disorders, the evidence was inconclusive, but favorable toward the use of manipulation and mobilization of the foot to decrease pain associated with Morton's neuroma ${ }^{(16)}$. Another study agreed that it was possible manipulation and mobilization provided short-term relief as a treatment of Morton's neuroma, but drew no definitive conclusion $^{(17)}$. Research focused on other areas of Morton's neuroma treatment, as well as many medically affiliated websites, have also suggested that local massage provides relief from pain $(2,9,10,12,13,14)$.

\section{Case Report Objective}

Surprisingly few case reports and research studies are available that focus on the effect of manual softtissue manipulation by a health care professional to manage pain associated with Morton's neuroma. Despite the high incidence of Morton's neuroma, reviews of current research in 2004 and 2007 concluded that there is insufficient evidence in randomized control trials to determine the efficacy of either surgical or nonsurgical interventions ${ }^{(17,18)}$. While more research has been conducted since these reviews, Morton's neuroma continues to be a complex condition with a wide variety of conservative and surgical interventions. The objective of this case report is to describe the effect of massage therapy on one client's pain symptoms associated with Morton's neuroma over the course of six weeks of massage once a week and a daily home care exercise. While the findings will only describe the efficacy of massage with one person, this massage therapist hopes it will add to the literature leading to a larger and more comprehensive study of massage and Morton's neuroma.

\section{METHODS}

\section{Client Profile}

The client is a 5 foot 10 inch, 145-pound, 25-yearold female with symptomatic Morton's neuroma between the third and fourth metatarsals in her left foot as diagnosed by her trauma doctor after X-rays to exclude a foot fracture. The client is physically active; she is an avid runner, swimmer, and biker who exercises four to six times per week. She competes in running races ranging in length from $5 \mathrm{~K}-12 \mathrm{~K}$. She considers her job to be stressful, and she typically exercises for stress reduction. At the time of starting the massage therapy treatments, she had been unable to run for the three months prior due to pain. Her job requires many hours on her feet, setting up outdoor research projects and lifting heavy objects.

Upon diagnosis with Morton's neuroma, the client's physician recommended rest (one month with no exercise) and a cortisone injection. The client complied by receiving the shot and ceasing running, but was unable to be off of her feet at work. After one month of inactivity and the cortisone shot, the pain had lessened slightly, but was still persistent, both during activity and while resting. The client wears store-bought arch support at work and in her running shoes on a daily basis. She does not wear tight shoes or high heels. Relevant medical history includes Achilles tendonitis in the right foot seven years prior, as well as a fracture in the right foot six years prior. Her left foot pain is worsened by running, jumping, and squeezing the foot. During the course of massage treatment, the client continued to wear store-bought arch support, but received no other care for the condition.

A postural assessment in the first session revealed a moderately elevated left hip and left shoulder. She had moderate bilateral protraction of the scapulae and severely rounded shoulders, as well as moderate forward head posture. The client had no conditions which contraindicated massage. She reported consistent burning, throbbing, stabbing, and aching foot pain, both during activity and when resting. The orthopedic test for Morton's neuroma, squeezing inward from the medial and lateral sides of the metatarsals ${ }^{(5)}$, resulted in pain consistent with Morton's neuroma between the third and fourth metatarsals in the client's left foot. This result was consistent with the physician's diagnosis. The orthopedic test for Morton's neuroma was applied by the massage therapist as a teaching tool and acknowledgement of how this condition could be tested for without a doctor's diagnosis.

The client's stated goal for the sessions was to find relief from the Morton's neuroma pain and return to her physically active lifestyle.

\section{Treatment Plan-Intervention}

\section{Treatment Overview}

The massage therapy intervention consisted of the orthopedic test for Morton's neuroma and visual and palpation postural assessment, followed by six treatments with postural assessment at the start and end of each session. The client received treatment once a week for 60-75 minutes over the course of six consecutive weeks. In each session, the time was divided as follows: 5 minutes of visual and palpation postural assessment, 30 minutes of general postural realignment using various massage therapy techniques specific to the postural assessment findings, 30 minutes of treatment massage therapy to the foot and leg, and 5 minutes of reassessment.

\section{Massage Intervention-Postural Alignment}

People with Morton's neuroma may develop modified gaits or change functional movement to avoid placing weight on the most painful parts of the foot. The client may have first developed a tendency to rely 
heavily on the left foot years earlier when her right Achilles tendon was inflamed, and then a year later she broke her right foot. With the onset of Morton's neuroma pain, the client may have developed a second gait modification to take pressure off of the pain in her left foot. These modifications can effect body alignment, starting with the foot and ankle and leading to subsequent compensation patterns which progress up the entire body. Improper alignment can then place more stress on the symptomatic Morton's neuroma.

The literature reviewed in this report about Morton's neuroma cites only mobilization and manipulation of areas local to the Morton's neuroma pain $^{(1,2,9,10,12,13,14,16,17)}$. There was no research available to set a precedent for addressing postural alignment as a means to creating a change in the pain associated with Morton's neuroma. In a holistic view of the body, where the body is seen as an interconnected whole, a presenting symptom is treated not only at the area of pain, but also in the areas of misalignment and irregularity that may be contributing to the pain. When the myofascial relationships in the body shift, there are both "local and distant effects on the body-not just on muscles and fascia, but also on nerves and vasculature"(5). Postural alignment was addressed with this client to create better balance and structural integrity in an effort to reduce the negative effect of repetitive motion and poor body alignment, as these increase friction and stress on the Morton's neuroma. Treating the client's muscular imbalances was an important step in combating compensation patterns the client had adopted to avoid painful motions of the foot.

The postural section of the session focused on encouraging the client's alignment toward neutral through myofascial release using a combination of myofascial trigger point therapy, longitudinal stroking, and facilitated stretching to balance functional postural dysfunction of the hips and shoulder.

Individual techniques were chosen using the evidence informed practice model. The massage therapist took into consideration desired treatment and effect as described in Rattray and Ludwig, (5) personal experience, and methods to most effectively create change in the client's body. Techniques for this specific client focused on facilitated stretching of the quadratus lumborum and trigger-point therapy to the left gluteus medius to create a level pelvis, facilitated stretching of the pectoralis major, as well as facilitated stretching and longitudinal stroking of the bilateral upper trapezius and levator scapula to balance the shoulder girdle.

\section{Massage Intervention-Local Foot and Leg Treatment}

Foot and leg massage therapy was implemented based on the suggestions in other studies that local massage provided relief from pain ${ }^{(1,9,10)}$. Since many muscles of the leg have attachment sites in the foot, both the smaller intrinsic foot muscles and the larger muscles of the leg with foot insertion sites were treated. The specific foot and leg treatment protocol was derived from the section on "Lower Extremity and Foot Dysfunction" in the bodywork manual for the Lumbar and Lower Extremity module of Boulder College of Massage Therapy's Orthopedic and Sports Massage Certificate Program ${ }^{(19)}$. These techniques included myofascial release and trigger-point therapy to the tibialis posterior, flexor hallucis longus, and flexor digitorum longus, longitudinal stroking of the gastrocnemius and soleus, passive calf, and Achilles tendon stretches, and cross-fiber friction of fibularis longus, gastrocnemius, and soleus attachments.

\section{At-home Exercise}

The client was provided an at-home exercise to do several times each day, focused on returning integrity to the deep transverse metatarsal ligament. Physical therapy suggestions, as well as research that advocates correcting ligament laxity and mechanical abnormalities, led the massage therapist to believe a daily at-home exercise would help progress continue between massages ${ }^{(6,8,14)}$.

The client was asked to sit with legs extended in front of the body and then to bring the metatarsals toward the shins and knees (dorsiflexion), without curling the toes (E.L. Calenda, oral communication, August 2010). The client performed the exercise first using her hand to place the foot in the proper position and then, as she became more skilled and gained fine motor control of the foot, she practiced without the aid of her hands (Figure 2).

The purpose of this exercise was to create more integrity in the deep transverse metatarsal ligament first by fostering the mind-body connection and establishing optimal foot position, and then by strengthening the actual muscular structures that maintain the optimal position.

\section{Treatment Plan-Assessments}

\section{Initial Intake}

The client filled out an extensive health history that included questions about how long she had been experiencing pain, treatment recommendations up to that point in time, whether or not she followed these recommendations, how often and what kind of exercise she engaged in, what she did for work, what shoes she wore, whether or not she wore shoe orthoses, previous injuries to either foot, hobbies that include time on her feet, and any activities that eased or worsened the pain. This questionnaire established the history, activities, and factors that may influence the pain associated with the client's Morton's neuroma.

\section{Orthopedic Assessment}

The orthopedic test for Morton's neuroma ${ }^{(5)}$, squeezing inward from the medial and lateral sides of the metatarsals, resulted in pain consistent with 

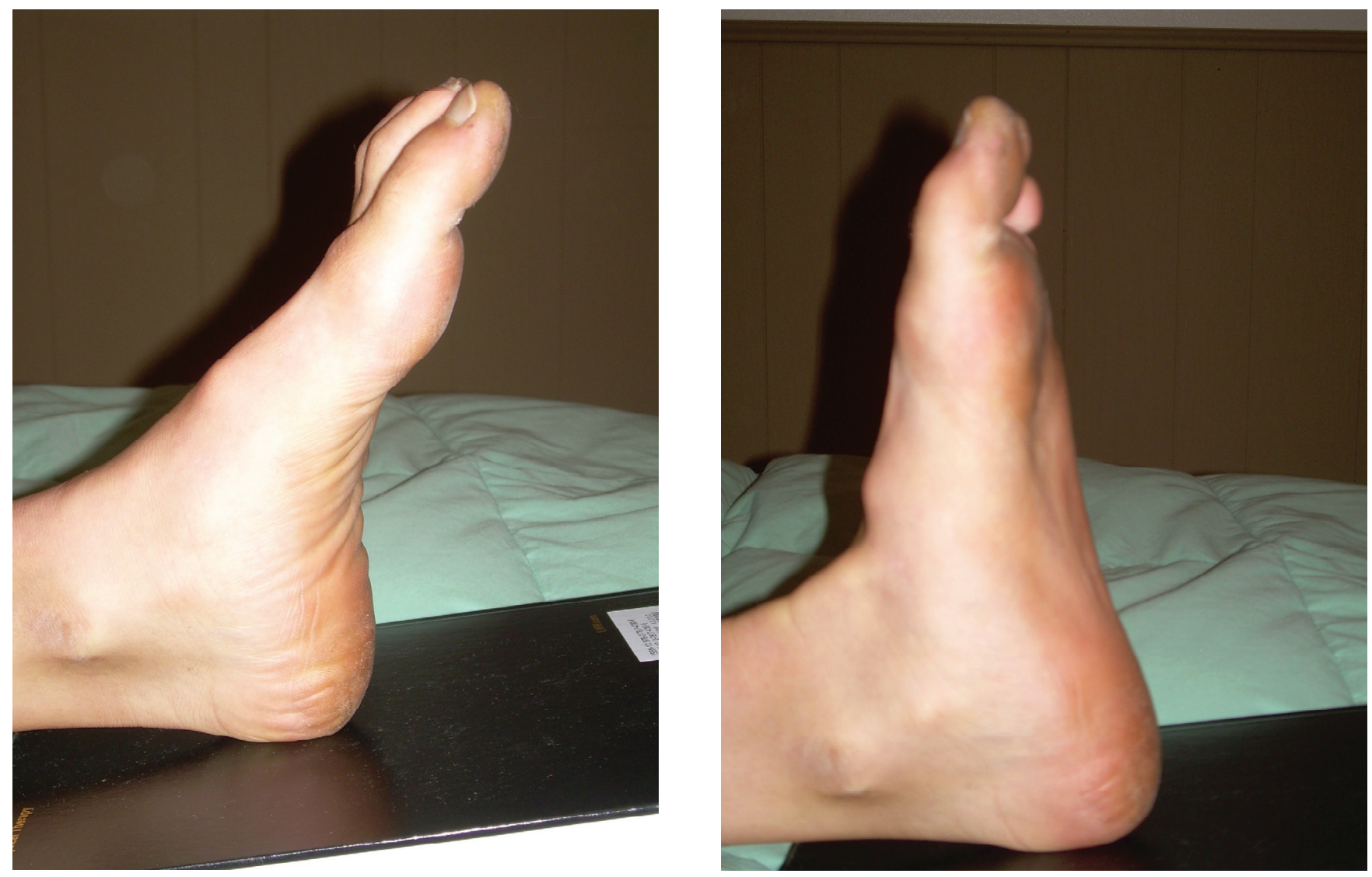

FIGURE 2. Client at Home Exercise. The left foot ( $a$, top) before deep transverse metatarsal ligament exercise. The left foot ( $b$, botton) during unassisted deep transverse metatarsal ligament exercise.

Morton's neuroma between the third and fourth metatarsals in the client's left foot. Although the test was unnecessary in this case because of the diagnosis as Morton's neuroma by a physician, it is included here as an educational piece for other massage therapists working with Morton's neuroma.

\section{Postural Assessment}

Before and after the massage at the first session and at each subsequent session, a complete visual and palpation postural assessment of the client was taken as she was standing, from both head-on and side views. Structural alignment and rotation of shoulders and hips and subsequent muscular imbalances were assessed visually by the massage therapist. Postural misalignment was assessed in relation to anterior, posterior, and lateral pelvic tilt, as well as spinal rotation and scapular imbalance. The massage therapist then palpated the same bony landmarks that were used in the visual assessment (anterior-superior iliac spine, posterior-superior iliac spine, and iliac crest and inferior and superior scapular angles) to confirm visual findings. These imbalances in the body were assessed because structural misalignment can exacerbate foot irritation by creating undue stress on particular areas, especially during repetitive motion.

\section{Visual Analog Scale}

Describing the change in pain symptoms associated with Morton's neuroma in relation to massage therapy is the primary concern of this case report. This made it essential to give the client a way to subjectively measure her experience of the changes. The Visual Analog Scale (VAS) allowed the client to measure her pain from week to week on a continuous scale. The VAS is particularly useful in a report where the scale is only describing one person's pain perception and would not be as applicable in a study with multiple clients because it does not offer a consistent measurement between people $^{(20)}$.

The client received three VASs at each session that were filled out during the course of the session. The first scale asked her to rate her average pain over the course of the week, the second asked her to rate her pain in the moment before beginning massage, and the third asked her to rate her pain at the end of the massage. Each scale went from zero to ten, with zero signifying no pain and ten signifying extreme pain. There was space provided at the end of the questionnaire for her to add any relevant qualitative changes in pain or variation in activity during the previous week. 


\section{RESULTS}

\section{Final Intake}

The client reported progressive change in the character of the pain from burning and stabbing before the first session, to a dull, pulsing sensation after the third session. She also reported a gradual decrease in the duration of pain after physical exercise. Further, the client was able to maintain decreased pain while increasing exercise and completing several short running races.

\section{Orthopedic Assessment}

The foot squeeze test was not repeated at the end of the massage series because the goal of the sessions was not to eliminate the Morton's neuroma, but rather to notice the changes, if any, in pain.

\section{Postural Assessment}

The client's alignment did not visibly change during the course of the sessions. Palpable changes in posture were limited. Following the sixth session, there was a decrease in the hypertonicity of the bilateral upper trapezius muscles and a release of trigger points in the left gluteus medius.

\section{Visual Analog Scale}

As shown in the graph (Figure 3), the client experienced a decrease in pain during physical activity, moving from five to zero on a scale of 1 to 10 (10 indicating the most pain). She also experienced a reduction in average pain while resting, pain at the moment of the start of the massage, and pain after the massage.

\section{DISCUSSION}

The objective of this case report was to describe the effect of massage therapy on one client's pain symptoms associated with Morton's neuroma in response to six weeks of weekly massage and daily home care exercise. The treatment series was effective for this client, particularly in reducing the pain during and

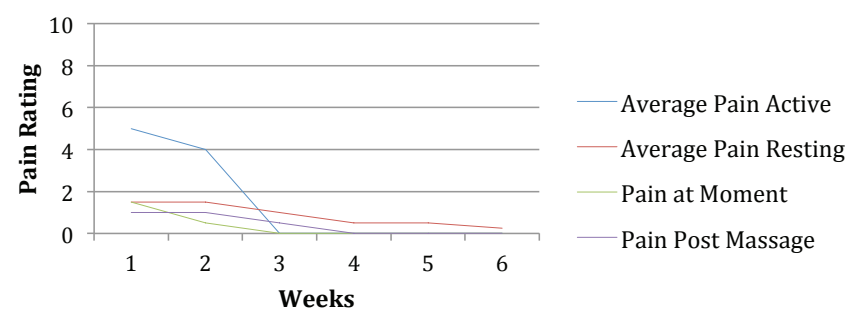

FIGURE 3. Pain Measured Over Time. after exercise, which allowed her to return to her usual activities. The improvement in pain could be the result of the multifaceted treatment plan which consisted of massage, continued rest without exercising during the first three weeks of massage, and compliance with the home care exercise which created a more stable environment in the foot. Each of these treatments has a similar effect in helping clients reduce pain, according to anecdotal evidence and other case reports $(1,2,9,10,12,13,14,16,17)$. There was no deviation from the treatment plan worth noting. Client and therapist were both vigilant in maintaining consistency of sessions and daily repetition of the at-home exercise. Pain reduction and the client's return to a normal level of activity may have contributed to decreased stress which, in turn, would help reduce pain.

\section{Measurement Tools}

The addition of a variety of objective measurement tools including muscle strength testing, the use of a goniometer, and evaluation based on established pain and mobility scales in future case reports may strengthen the consideration of massage therapy as an intervention for pain associated with Morton's neuroma. Assessing posture with a grid and plum line ${ }^{(21)}$ would allow precise measurement of postural imbalances, and would facilitate noticing small, measurable - but not visually perceivablechanges. A goniometer would be useful to create precise measurements rather than visual estimates. Individual muscle strength testing ${ }^{(5,21)}$ of leg muscles would direct the massage therapist to work specific muscles and be able to retest the same muscles to find correlations, if any, between changes in muscle tone and strength and change in pain. Assessing range of motion at the initial session, half way through, and at the final session would also give a good measurement of changes in foot and ankle mobility.

Using previously established scales would allow future reports to be easier to replicate. The use of an established scale for measuring muscle tone when palpating would be necessary, especially in a larger scale study involving multiple clients and therapists. An established scale has the benefit of being more precise than the descriptors mild, moderate, and severe. The Foot Function Index as a means of measuring foot pain and disability only came to the attention of this massage therapist after completion of the case report $^{(22)}$. This scale would be an excellent and replicable tool for measuring and comparing pain and activity changes between individual clients.

\section{Recommendations for Future Studies}

The results of this case report are not conclusive and warrant further investigation to validate massage treatment as an adjunctive or principal option for treatment of Morton's neuroma. Future case reports 
using the additional measurement tools as previously mentioned would be the first step to creating a body of literature measuring the efficacy of massage in reducing pain associated with Morton's neuroma. Larger studies could then be done to demonstrate statistical significance of massage treatment.

Determining the impact of postural alignment on Morton's neuroma requires a study of much longer duration, and faces the challenge of working with individual postural misalignment under controlled conditions.

In this report, once the client's pain had been moderately reduced in the first three weeks of treatment and she was able to return to exercise, her pain continued to decrease during the final three weeks of treatment. In future studies, tracking the link between decreased pain and improved quality of life would help to establish either a direct or indirect connection between the two.

In this case report, leg and foot massage seemed more effective than postural alignment because so little alignment change was observed. A two-armed study (leg and foot massage only, leg and foot massage combined with postural alignment) might relate the efficacy of these two methods.

\section{CONCLUSION}

Morton's neuroma is a condition of the forefoot which, when symptomatic, causes pain that limits mobility and regular daily activity. Many conservative and surgical interventions are employed to reduce associated pain, but not enough research has been conducted to recommend patients to any one approach as the most reliable source of pain management. This study describes how massage therapy can play a role in reducing pain from Morton's neuroma; however, larger randomized control studies need to be done to determine the short- and long-term effects of massage therapy on this painful condition.

\section{ACKNOWLEDGEMENTS}

Many thanks to my case report supervisor Elaine Calenda, RMT, AOS who listened to my questions, ideas, and often helped to steer me back on course. A heartfelt thanks to friends, colleagues, and editors who willingly shared their time and knowledge as I wrote this case report. My gratitude also to the Massage Therapy Foundation which recognized this report with a bronze award in the 2011 Student Case Report Contest.

\section{CONFLICT OF INTEREST NOTIFICATION}

The author declares there are no conflicts of interest.

\section{COPYRIGHT}

Published under the CreativeCommons AttributionNonCommercial-NoDerivs 3.0 License.

\section{REFERENCES}

1. Bencardino J, Rosenberg ZS, Beltran J, et al. Morton's neuroma: is it always symptomatic? AJR. 2000;175(3):649-653.

2. Berry K, Gonzalez P, Bowman RG. Physical Medicine and Treatment for Morton Neuroma. Available from: http:// emedicine.medscape.com/article/308284-overview. Updated March 30, 2012. Accessed January 25, 2012.

3. Summers A. Diagnosis and treatment of Morton's neuroma. Emerg Nurse. 2010;18(5):16-17.

4. Hughes, RJ, Ali K, Jones H, et al. Treatment of Morton's neuroma with alcohol injection under sonographic guidance: follow-up of 101 cases. AJR. 2007;188(6):1535-1539.

5. Rattray F, Ludwig, L. Clinical Massage Therapy: Understanding, Assessing And Treating over 70 Conditions. Elmira, ON: Talus Incorporated; 2000.

6. Spina R, Cameron M, Alexander R. The effect of functional fascial taping on Morton's neuroma. Australas Chiropr Osteopathy. 2002;10(1):45-50.

7. Quinn TJ, Jacobson JA, Craig JG, et al. Sonography of Morton's neuromas. AJR. 2000;174(6):1723-1728.

8. Kay D, Bennett GL. Morton's neuroma. Foot Ankle Clin. 2003;8(1):49-59.

9. Peng H, Swierzewski SJ III. Morton's Neuroma. Available from: http://www.healthcommunities.com/mortons-neuroma/ about-mortons-neuroma.shtml. Published December 31, 1999. Updated May 16, 2011. Accessed January 26, 2012.

10. Guiloff RJ, Scadding JW, Klenerman L. Morton's metatarsalgia. Clinical, electrophysical and histological observations. J Bone Joint Surg Am. 1984;66-B(4):586-591.

11. Sharp RJ, Wade CM, Hennessy MS, et al. The role of MRI and ultrasound imaging in Morton's neuroma and the effect of size of lesion on symptoms. J Bone Joint Surg Am. 2003;85B(7):999-1005.

12. American College of Foot and Ankle Surgeons. Morton's Neuroma. Available from: http://www.foothealthfacts.org/ footankleinfo/mortons-neuroma.htm. Updated August 23, 2010. Accessed September 16, 2010.

13. Mayo Clinic. Morton's Neuroma. Available from: http://www. mayoclinic.com/health/mortons-neuroma/DS00468. Published \& Updated October 5, 2010. Accessed September 16, 2010.

14. PhysioAdvisor. Morton's Neuroma. Available from: http:// www.physioadvisor.com.au/13489750/mortons-neuromametatarsalgia-physioadvisor.htm. Published 2008. Accessed Jan 24, 2012.

15. Joswick D. Treating Morton's Neuroma with TCM. Available from: https://www.acufinder.com/Acupuncture+Information/ Detail/Treating+Morton's+Neuroma+with+TCM. Published 2012. Accessed January 24, 2012.

16. Bronfort G, Haas M, Evans R, et al. Effectiveness of manual therapies: the UK evidence report. Chiropractic \& Osteopathy. 2010;18(3):1-33. Available from: http://www.biomedcentral.com/ content/pdf/1746-1340-18-3.pdf. Accessed January 24, 2012. 
17. Govender N, Kretzmann H, Price JL, et al. A single-blinded randomized placebo-controlled clinical trial of manipulation and mobilization in the treatment of Morton's neuroma. J Amer Chiropr Assoc. 2007;44(3):9-18.

18. Thomson CE, Gibson JN, Martin D. Interventions for the treatment of Morton's neuroma. Cochrane Library. 2009. Available from: http://onlinelibrary.wiley.com/doi/10.1002/14651858. CD003118.pub2/abstract.

19. Quinn C, Morgareidge K, Chandler C, et al. Lumbar and Lower Extremity Treatment Manual. Boulder, CO: Boulder College of Massage Therapy. Orthopedic and Sports Massage Certificate Program; 2006.

20. Crichton, N. Visual analog scale (VAS) [Information Point]. $J$ Clin Nurs. 2001;10:706.
21. Kendall FP, McCreary EK, Provanve PG, et al. Muscles: Testing and Function, with Posture and Pain, 5th edition. Baltimore, MD: Lippincott Williams \& Wilkins; 2005.

22. Budiman-Mak E, Conrad KJ, Roach KE. The foot function index: a measure of foot pain and disability. J Clin Epidemiol. 1991;44(6):561-570.

Corresponding author: Faith Davis, BA, RMT, NCTMB, AOS, Private Practice, 726 C Pearl St., Boulder, CO 80302, USA

E-mail: faith.davis@me.com 